\title{
Le français, la Francophonie et l'interculturel
}

\author{
Julio E. Sánchez Murillo' \\ Universidad Nacional, Costa Rica
}

\section{RESUMEN}

La expansión colonialista de Francia y la presencia del francés en la gran mayoría de los sistemas educativos contribuyeron de sobremanera a la difusión de esta lengua a través del mundo. Estos hechos facilitaron la fijación de una imagen estereotipada de Francia, de los franceses y de la misma lengua francesa. Hoy, la Francofonía y la enseñanza del francés tienen como objetivos primordiales, entre otros, liberar el francés de sus viejos estereotipos, dar una dimensión internacional de esta lengua y promover la diversidad cultural y lingüística y la comprensión entre los pueblos, esto en el marco de un enfoque intercultural.

\section{RÉSUMÉ}

L'expansion colonialiste de la France et la présence du français dans la plupart des systèmes éducatifs ont grandement contribué à la diffusion de cette langue dans le monde. Ces faits ont facilité la fixation d'une image stéréotypée de la France, des Français et même de la langue française. Aujourd'hui, la Francophonie et l' enseignement du français ont pour objectifs primordiaux, parmi d'autres, libérer le français de ses vieux stéréotypes, donner une dimension internationale de cette langue et promouvoir la diversité culturelle et linguistique et la compréhension entre les peuples, ceci dans le cadre d'une approche interculturelle.

$\overline{1}$ Correo electrónico: jesm80@ costarricense.cr 
Palabras clave: francés, enseñanza del francés, Enfoque intercultural, Francofonía.

Mots-clés : français, enseignement du français, Approche interculturelle, Francophonie.

Le français n'appartient plus aux seuls Français, mais à tous ceux qui, à un titre ou à l'autre, le parlent et l'utilisent dans toutes sortes de fonctions. Pierre Dumont

Aujourd'hui, le français est la langue d'un ensemble de peuples regroupés dans une organisation, l'OIF, qui promeut la paix, la démocratie, la diversité culturelle et linguistique, tout en donnant à la langue française le rôle d'un prestigieux instrument de communication. Grâce à l'enseignement de celle-ci à travers le monde et l'étude des cultures francophones, dans une perspective interculturelle. La lutte contre le monolinguisme et l'uniformisation des cultures est menée.

Cet article se propose de faire un bref rappel historique de l'expansion de la langue française et de la création de la Francophonie et une réflexion théorique sur l'importance de l'application d'une approche interculturelle à l'étude des cultures francophones dans le cadre de l'enseignement du FLE car celles-ci contribuent à la promotion de la pluralité culturelle et linguistique.

\section{L'expansion du français}

La langue française est née, entre le $\mathrm{VI}^{\mathrm{e}}$ et le $\mathrm{IX}^{\mathrm{e}}$ siècles, grâce au contact des Romains avec des peuples gaulois, celtes et germaniques. Avec le temps, le français est devenu la langue d'un des pays les plus influents du monde. Au Moyen-Âge, période d'apogée de la France (le pays le plus peuplé mais aussi le plus riche d'Europe), la langue française a pris de plus en plus importance, comme en témoignent certains faits : le français s'est répandu au 102 
Proche-Orient, comme une conséquence des croisades répondant à l'appel du pape Urbain II ( $\mathrm{XI}^{\mathrm{e}}$ siècle) qui envisageait la reconquête des lieux saints chrétiens. En outre, les royaumes d'Antioche et de Jérusalem, créés par des croisés français, ont adopté le français comme langue officielle.

Son rayonnement en Europe se poursuit dans le domaine commercial puisque le français est devenu, à la fin du Moyen-Âge et aux XII ${ }^{e}$ et XIII ${ }^{e}$ siècles, la langue internationale des transactions et des échanges commerciaux. En outre, les universités de France étaient les plus renommées du Vieux Contient et attiraient de nombreux étudiants des quatre coins de l'Europe. Ceci a contribué à répandre, non seulement, le français mais aussi une certaine vision du monde «à la française ».

Malgré la suprématie de cette langue, vers la fin du XVI siècle, celle-ci s'est vue concurrencée par l'espagnol et surtout par l'italien. Toutefois, cette concurrence n'a été que temporaire. En effet, grâce à l'essor de la France pendant le règne de Louis XVI, la langue française a repris sa place. À partir de cette époque-là, elle a acquis un grand prestige et est devenue la langue de la noblesse européenne et de la diplomatie. Les enfants des rois et des princes étaient élevés en français et par des précepteurs français.

Cette éducation à la française se répandait en Europe comme un idéal qui dominait les élites. Durant ces siècles, le français et les « bonnes manières » marchaient la main dans la main. L'enseignement consistait, surtout, en l'apprentissage de la langue, mais aussi, en l'appropriation des normes, des comportements et des pratiques liées à la vie sociale. Dans l'enseignement du français, on utilisait comme supports pour développer les cours, la grammaire, le dictionnaire et les textes littéraires car ceux-ci étaient « en eux-mêmes des lieux où la civilisation française se manifeste de façon directe ou indirecte, dans les choix de vocabulaire, de mises en scène pour présenter les règles grammaticales, de thèmes pour les exercices ${ }^{2}$.

\footnotetext{
${ }^{2}$ M. C. Kok-Escalle, "Civilisation française : de la langue à la culture », Le français dans le monde, numéro spécial (1998) : 173.
} 
Vers la fin du XVIII et le début du XIX ${ }^{e}$ siècle, deux événements ont contribué à l'expansion du français. Premièrement, le processus de colonisation et puis, l'élargissement de l'enseignement de cette langue au secondaire, ce qui a permis à un grand nombre d'adolescents de plusieurs pays du monde de mettre en contact avec le français. L'enseignement qu'on proposait à cette époque-là menait à l'enrichissement de l'esprit au moyen de la découverte par l'apprenant d'une « civilisation supérieure et évoluée » de l'humanité à travers la littérature. L'œuvre littéraire, comme support, permettait d'enseigner la langue à l'aide de textes hors pair et qui pouvaient être pris comme des modèles à suivre lors des productions des apprenants.

Cette idée de «culture supérieure et évoluée » et la « mission civilisatrice », que la France s'est attribué, ont justifié et soutenu, en quelque sorte, le processus de colonisation qu'elle venait d'entreprendre. La colonisation, dès la perspective européenne, permettait aux peuples des autres régions, dénommés à l'époque comme sauvages et barbares, d'approcher leur civilisation. De cette façon, ces sociétés ont imposé, violemment, leur vision du monde en mettant en relief leur progrès scientifique et technique, leur production et leur mode de vie aux groupes sociaux habitant les pays qui sont devenus des colonies européennes (françaises, anglaises, espagnoles, portugaises, entre autres) au fur et à mesure que le XVIII et le $\mathrm{XIX}^{\mathrm{e}}$ siècles avançaient ; tout cela au nom de "la progression de l'humanité ».

On ne doit pas oublier un autre fait important : la langue française était parlée par l'équipage des navires des explorateurs, des conquérants et des colons qui sont arrivés à diverses régions qui ont été rapidement annexées à l'empire colonial. De manière succincte, on vient de voir que deux faits majeurs ont marqué l'expansion du français : la colonisation et l'enseignement de cette langue tant dans les colonies que dans les pays indépendants et par conséquent, le français est devenu une langue à dimension internationale. 


\section{La Francophonie ${ }^{3}$}

Le processus de colonisation a mené à la structuration d'un ensemble de territoires sous la domination française. Ceci a permis au français de s'implanter un peu partout dans le monde. Au fur et à mesure que la colonisation avançait, l'enseignement du français s'est disséminé. À cette période-là, il était encore la langue des élites mais sa progression dans d'autres couches sociales était notoire. Il est à remarquer que le français est devenu, dans les colonies, la langue de l'administration, de l'enseignement, du travail, de la presse, entre autres ; une situation qui a contribué, définitivement, à sa propagation.

En 1880, en plein apogée de l'Empire colonial, le géographe français Onésime Reclus a créé le terme francophonie. Contrairement à la mentalité coloniale de l'époque, il prenait comme critère de classement, non pas la position de domination ou de dépendance, mais la langue parlée au quotidien. Ainsi, la notion de francophonie faisait référence à l'ensemble des espaces où l'on parlait français. Néanmoins, ce mot n'a pas eu beaucoup de diffusion et a resurgi, modestement, dans les dictionnaires vers 1930.

L'Empire colonial s'est consolidé dans les premières décennies $\mathrm{du} \mathrm{XX}^{\mathrm{e}}$ siècle. La France dominait un territoire de 10 millions de $\mathrm{km}^{2}$ et peuplé de 50 millions d'habitants. Mais, le début de ce siècle a marqué aussi la fin de la suprématie de la langue française, ceci aux dépens de l'anglais. À cette époque-là, une image de la France, des Français et de la langue française, parfois très stéréotypée, s'était bien fixée, ceci grâce, en grande partie, à l'enseignement du français. Après la Seconde guerre mondiale, le processus de décolonisation s'est déclenché et par conséquent, le démantèlement des empires coloniaux puisque les divers mouvements indépendantistes et nationalistes cherchaient l'émancipation des peuples soumis au pouvoir européen.

\footnotetext{
${ }_{3}$ Remarque : francophonie (avec une minuscule initiale) fait allusion à l'ensemble des peuples ou des groupes de locuteurs qui utilisent le français dans leur vie quotidienne ou leurs communications. Francophonie (avec une capitale initiale) désigne l'ensemble des gouvernements ou instances officielles qui font partie du projet politico-culturel de l'Organisation internationale de la Francophonie.
} 
Au fil des années, la plupart des pays qui composaient l'Empire, ont obtenu leur autodétermination de manière relativement pacifique ; à l'exception de l'Indochine (1946-1954) et de l'Algérie (1954-1962), qui ont accédé à l'indépendance après une douloureuse guerre. Dans les années 1960, l'ancien grand Empire colonial français s'est réduit à quelques îles dispersées sur les principaux océans du monde (Pacifique, Atlantique et Indien). Cependant, la colonisation a laissé des traces profondes dans les nouveaux États, parmi les plus importantes : la langue française, soit comme langue officielle, soit comme langue privilégiée d'enseignement.

Malgré les plaies causées par la longue période de soumission coloniale, les années qui ont suivi l'indépendance se sont caractérisées par la résurgence d'un mouvement cherchant le rassemblement des peuples autour de la langue française. En outre, cette époque-là a marqué la véritable renaissance du terme francophonie. En 1962, dans la revue Esprit, Léopold Sédar Senghor y a créé le concept moderne : « la francophonie, c'est cet humanisme intégrale qui se tisse autour de la terre, cette symbiose des énergies dormantes de tous les continents, de toutes les races qui se réveillent à leur chaleur complémentaire ${ }^{4}$.

Petit à petit, la future organisation de la Francophonie a commencé à prendre force grâce, principalement, à l'impulsion de Senghor (Sénégal), Hamani Diori (Niger) et Habib Bourguiba (Tunisie) ; outre le notable soutien du prince de Cambodge Norodom Sihanouk et du président libanais Charles Héloun. De nombreuses associations francophones ont vu le jour au lendemain de l'indépendance des anciennes colonies. Ce processus associatif a mené, malgré certaines réticences de la France, à la création en 1970, à Niamey (Niger) de la première structure intergouvernementale francophone : l'Agence de coopération culturelle et technique.

Au cours des décennies, cette institution est devenue l'Organisation internationale de la Francophonie (OIF) et regroupe, à

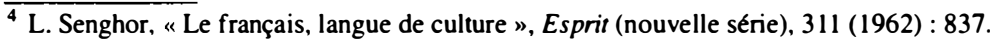


présent, 68 États et gouvernements membres, associés ou observateurs convoqués tous les deux ans au Sommet de la Francophonie. Aujourd'hui, l'OIF rassemble des pays développés -France, Suisse, Canada, Belgique, entre autres, mais aussi des pays très défavorisés Haïti, Mali, Djibouti, par exemple.

Dans l'actualité, cette organisation promeut la diversité culturelle, le développement économique et scientifique et la solidarité entre les peuples. En outre, elle lutte contre l'uniformisation linguistique et prône le plurilinguisme tout en gardant, au français, un rôle prépondérant comme "langue en partage ». Pendant le processus de construction et consolidation de cette institution, rassembleuse de la quasi-totalité de pays et régions francophones, l'enseignement du français a subi des changements dans le but de l'améliorer, de le répandre et de faire face à la montée du dangereux monolinguisme.

Ce renouvellement s'est caractérisé par la mise au point d'une approche qui tient au développement non seulement des quatre compétences mais qui cherche aussi à présenter une langue française proche de la réalité quotidienne de ses locuteurs et qui peut être un excellent moyen pour s'insérer, avec succès, dans un monde contemporain marqué par les avances scientifiques, la culture vivante (musique, cinéma, etc.), le rôle prédominant des médias et les échanges commerciaux. D'ailleurs, dès cette nouvelle perspective, le français est un précieux outil qui favorise le contact avec une multiplicité de cultures des quatre coins de la terre. Ainsi, l'enseignement de cette langue essaye de se débarrasser de son image élitiste et démodée.

Etant donné que le français est la langue de 200 millions de personnes dans plus de 50 pays ou régions, que le multiculturalisme est soutenu par la France et tous les autres pays francophones et qu'un des objectifs de l'enseignement-apprentissage de langues est la connaissance mais surtout la compréhension de l'autre ; depuis quelques années, on a inclut dans les programmes de FLE ou FLS, l'approche interculturelle pour fomenter cette compréhension entre 
les peuples, si nécessaires à nos jours, et pour faciliter l'effacement des images stéréotypées de la France et des autres pays francophones. De cette manière, étudier les cultures francophones, dans le cadre de l'enseignement du français, ouvre une fenêtre à un monde différent, riche et divers.

À travers l'histoire de l'enseignement du français, l'étude des aspects culturels s'est focalisée sur la France et à partir de différentes approches, telles que : les approches descriptive, contrastive ou thématique. Cependant, ces approches menaient à une vision réductrice, simpliste, voire, stéréotypée de l'autre. En revanche, l'approche interculturelle cherche à présenter les diverses manif estations culturelles des Français et des francophones. C'est pour cela qu'il s'avère pertinent d'exposer en détail l'approche interculturelle et ses implications.

\section{L'approche interculturelle}

Indubitablement l'approche qui est de plus en plus considérée comme la plus adéquate pour enseigner une culture quelconque, est l'approche interculturelle. Cette approche apparaît au début des années 80, même si quelques spécialistes en parlaient depuis quelques années. Cette époque s'est caractérisée par le travail de renouvellement de l'enseignement de la culture dans l'univers du FLE. L'approche interculturelle fait partie de ce mouvement qui cherche à libérer l'enseignement du français, dans toutes ses composantes, à savoir la grammaire, l'oral, l'écrit, la littérature et bien sûr la culture ; des pratiques traditionalistes, mémorialistes et des pratiques qui essayaient de transmettre une image idyllique et élitiste de la langue française et de la France. L'approche interculturelle a surgi dans une période marquée par les valeurs de l'individualisme et de la compétitivité. Pour faire face à ces situations, cette approche travaille à la prise en compte de la diversité sociale et culturelle des sociétés faisant partie de ce qu'on a appelé la post-modernité. Ces sociétés sont caractérisées par le pluralisme. 
L'approche interculturelle comme base théorique, favorise des pratiques qui tendent à promouvoir une évolution positive des images de soi et d'autrui. En outre, elle cherche à introduire une réflexion des valeurs sur lesquelles se construit une société et à mener à la compréhension de celle-ci dans sa complexité et ses contradictions. L'interculturel est défini comme un phénomène de représentations en contraste, débouchant irrémédiablement sur des stéréotypes mais qui se construit comme un processus, un échange entre la culture maternelle et la culture étrangère ayant pour objectif la fin des barrières, la réciprocité et la solidarité entre peuples.

Travailler sous la perspective interculturelle implique un changement méthodologique. Cette perspective se construit à partir de certains axes dont le plus important est l'altérité. Celle-ci « constitue une facette de toute société comprenant plus d'un groupe ethnique, et habituellement par conséquent, plus d'une langue maternelle ${ }^{5}$. Ces deux situations se présentent dans les pays francophones plus clairement qu'au Costa Rica où presque la totalité de la population a comme langue maternelle l'espagnol. Dans le cadre de l'enseignement de langues étrangères, l'altérité constitue un moyen de protéger l'identité nationale puisqu'elle se base sur les différences. Dans ce même cadre, on peut mentionner comme apports de l'enseignement de langues, la présentation de ce qui est différent aux apprenants et une aide à la compréhension de l'autre. Il faut ajouter que les apprenants doivent confronter la langue des autres, leur culture, leur manière de penser et d'affronter le monde, des éléments caractéristiques de l'autre.

L'objectif de l'enseignement de la culture, dans le cadre de cette approche, est de développer une compréhension des cultures à partir d'une ligne de travail privilégiant la réflexion sur les hommes et leurs mœurs, leurs habitudes et leurs comportements c'est-à-dire l'expression d'une culture. Pour atteindre cet objectif, l'approche

$\overline{5}$ M. Byram, Culture et éducation en langue étrangère (Paris : Didier/Hatier, 1992) 45. 
interculturelle propose de développer le travail en classe à partir de différents moyens pour une meilleure lecture des cultures qui conduise à une compréhension. Cette compréhension doit être accompagnée d'une reconnaissance de l'autre, de cette manière l'étranger est présenté comme un être qui agit et pense différemment mais ces dissemblances par rapport à soi ne font pas de celui-ci un être inférieur ou supérieur.

S'initier à l'étude de l'autre est important car elle permet de rendre objective la vision qu'on a de la culture maternelle, « en tant qu'élément de la dualité, autrui est constitutif du sentiment d'identité, que celui-ci soit défini en termes psychologiques, culturels, ethniques... individuels ou collectifs ${ }^{6}$. C'est-à-dire, il existe une complémentarité entre soi et l'autre, celle-ci se construit dans une perspective interactionniste. Cette perspective conçoit les différences entre cultures comme un rapport active et égalitaire qui s'octroie une signification mutuelle. L'objectif n'est pas rendre absolues ces différences, c'est plutôt de les relativiser dans un contexte social, économique et historique déterminé.

Dans cette même voie, l'approche interculturelle propose une étude de la culture par le biais de trois termes à savoir, en termes de représentations, en termes de frontières et en termes de communication. On va se concentrer surtout sur l'étude en termes de représentations. Depuis quelques décennies, on considère que ce sont les individus qui conforment les cultures et non les cultures en soi ce qui sont vraiment en contact, c'est pour cela que l'étude des représentations qu' un groupe d'individus a d'un autre ou de soi-même est devenue importante. Moscovici définie une représentation comme " une organisation psychologique, une modalité de connaissance particulière...la représentation se définit en premier lieu comme un processus de médiation entre concept et perception..., processus qui

${ }^{6}$ M. Abdallah-Preteceille, " La perception de l'autre », Le français dans le monde, 181 (1983) : 42. 
rend en quelque sorte le concept et la perception interchangeable du fait qu'ils s'engendrent réciproquement ${ }^{7}$.

Les représentations évoluent avec les individus et le temps. De cette façon, on évite l' enfermement de soi ou des autres dans certaines idées. En plus, l'étude des représentations permet d'avoir des informations très utiles autant sur ceux qui les émettent que sur ceux qui sont l'objet du jugement des autres. On parlera ultérieurement de l'importance de l'étude de celles-ci.

Après toutes ces considérations générales sur l'approche interculturelle, il convient maintenant de se centrer dans l'application de celle-ci dans une classe de FLE. Premièrement, l'approche interculturelle met l'accent sur l'appréhension d'une unité de valeurs différentes et sur l'analyse des manifestations de la culture étrangère ainsi que sur la culture maternelle permettant la prise de conscience de l'importance de la relativité, des implicites culturels et de l'ethnocentrisme. En outre, l' approche interculturelle se distingue des autres approches par ses objectifs, les contenus à développer et les démarches d'étude. Il est essentiel de retenir ces aspects pour la mise en place de l'approche interculturelle.

Le statut de l'apprenant est un élément à être pris en considération lors de l'application de cette approche car ceci pourrait faciliter l'ouverture à l'autre et la disponibilité à l'étude d'une culture étrangère. G. Zarate ${ }^{8}$ établit quatre statuts différents des étudiants qu'il faut considérer :

- ceux qui ont déjà étudié ou initié l'apprentissage d'une langue étrangère (ces apprenants ont déjà une expérience interculturelle)

- des apprenants bilingues pour diverses circonstances (ils ont vécu l'expérience interculturelle)

- des apprenants qui ont fait un séjour à l'étranger

\footnotetext{
${ }^{7}$ Abdallah-Preteceille, 43.

8 Geneviève Zarate, " Du dialogue des cultures à la démarche interculturelle ", Le français dans le monde, 170 (1982) : 31 .
} 
- ceux qui ont une expérience interculturelle grâce à une relation individuelle ou à une relation de famille. Ces relations peuvent se présenter avec un étranger ou avec une personne appartenant à une autre classe sociale et peuvent être déterminées par la durée de celles-ci.

Dans le cas de l'enseignant, ces situations pourraient aussi se présenter. Cependant son cas est différent, il est possesseur d'un savoir sur une culture autre et par conséquent, un informateur privilégié, mais il est surtout un intermédiaire entre la culture maternelle et la culture étrangère. Dans certains cas, le professeur a tendance à imposer, à ses étudiants, ses propres représentations de la culture cible. De cette façon, l'étude de cette culture se restreint à la subjectivité de celui-ci. D'autres risques à éviter sont : l'infériorisation de la culture autre ou la valorisation démesurée de celle-ci en la présentant comme supérieure à la maternelle.

Un autre élément important de cette approche sont les manifestations culturelles et interculturelles, celles-ci deviennent des objets pour l'étude de la culture. D'abord, il faut signaler la différence entre celles-ci. Les objets culturels sont des « traces de l'imaginaire psychosocial ${ }^{9}$.

Ces objets font partie de « l'ensemble de pratiques sociales et d'un ensemble de discours construits sur ces pratiques $»{ }^{10}$ tels que :

- l'organisation de l'espace quotidien

- la structuration du temps

- la place du corps dans l'espace social

- les comportements et les attitudes des gens

- la parole

- les représentations-emblèmes

9 P. Charaudeau, "L'interculturel, une histoire de fou », Dialogues et cultures, 32 (1988) : 94.

${ }^{10}$ M. B. Le Berre, Compétence culturelle et didactique des cultures (Poitiers : CNED, 1992) 77. 
Les objets de l'interculturel sont constitués des représentations contrastées. Il existe deux façons de les étudier :

- faire l'inventaire des représentations, qui selon M. B. Le Berre, aboutissent à une liste de stéréotypes

- découvrir les supports de ces jugements et justifier ceux-ci

Ces objets peuvent être envisagés dans une démarche interculturelle consistant à confronter les apprenants à des inf ormations de la culture étrangère et en langue étrangère le plus contextualisées et authentiques possible. Selon $\mathrm{H}$. Besse ${ }^{11}$, ces informations doivent présenter une certaine étrangeté pour les apprenants dans le but d'éveiller leur curiosité et provoquer un regard sur la culture maternelle. Le deuxième pas serait d'encourager les étudiants à formuler des hypothèses interprétatives des informations présentées. Cette formulation peut se faire en langue maternelle ou en langue cible ; dans notre cas, il serait préférable de l'exprimer plutôt en français.

Ces hypothèses reflètent la compréhension et l'incompréhension de la culture autre. En outre, elles montrent à l'enseignant les représentations et les stéréotypes que les apprenants ont de la culture à étudier. L'enseignant doit indiquer et expliquer si les hypothèses sont valables ou pas. Cette interaction entre culture maternelle/culture étrangère et enseignant/apprenant permet à ceux-ci de découvrir non seulement que leur pensée peut être d'une certaine manière semblable ou différente de celles des étrangers ; mais aussi la perception des individus et des choses « est en partie une reconstruction culturelle du monde et que cette reconstruction n'est pas identique à celle impliquée par la langue/culture étrangère ${ }^{12}$. Cette proposition est un exemple intéressant d'une démarche interculturelle qui, même si elle a été proposée il y a quelques années, est toujours valable et

"H. Besse, "Éduquer la perception interculturelle », Le français dans le monde, 188 (1984) : 49

12 Besse, 49. 
pourrait contribuer au développement de l'interculturel dans les classes de FLE ou servir comme point de départ pour la formulation de nouvelles démarches.

Il serait important de retenir la réflexion de $\mathrm{M}$. AbdallahPretceille, à propos de cette approche : "l'approche interculturelle remettant ainsi à l'honneur la subjective (qu'il ne faut pas confondre avec la subjectivité), l'éphémère, l'hétérogène, l'aléatoire, la complexité et l'incomplétude... qui sont autant d'expressions de l'humain. Il s'agit d'un type de lecture des cultures, qui loin de revendiquer une quelconque suprématie sur les autres approches se présente comme un point de vue, un choix, assumé non pas à partir de la négation des autres, mais par un souci d'ancrage des échanges langagiers dans une sociologie et une anthropologie compréhensives et non pas seulement explicatives du quotidien, en évitant de sombrer dans une approche réductionniste par catégorisation a priori et donc hors contexte du comportement communicatif d'autrui ${ }^{13}$.

Dans le cadre d'une approche interculturelle, il s'avère indispensable une étude des représentations et stéréotypes qu'on a de la culture cible et des individus qui la conforment puisqu'ils deviennent évidents plus facilement chaque fois qu'on entre en contact avec l'étranger. On doit aussi prendre en compte le fait, que pour beaucoup de gens, l'étranger et sa culture sont des objets fixes et facilement perceptibles. C'est pourquoi, cette étude est un passage obligé dans l'enseignement d'une culture étrangère car elle contribue à atteindre un des objectifs primordiaux de cet enseignement : la compréhension de l' autre. Proposer un travail sur les stéréotypes et les représentations est toujours valable quand il s'agit de faire acquérir une compétence interculturelle visant à donner l'image du ou des pays de la langue cible la plus proche possible de la réalité et loin des représentations folklorisantes d'une autre époque.

\footnotetext{
${ }^{13}$ M. Abdallah-Pretceille, "Compétence culturelle, compétence interculturelle ", Le français dans le monde, numéro spécial (1996) : 38.
} 
Il est pertinent de considérer certaines notions conceptuelles qui ont toujours été liées aux stéréotypes. Ces notions comprennent les représentations et les préjugés. La notion de représentation est souvent présentée avec les termes d'image, de regard ou de point de vue. Certains spécialistes considèrent ceci constitue une vulgarisation du terme. D'autres, la plupart peut-être, estiment que la manière de nommer ce concept n'est pas très importante comme le concept en soi-même.

Étymologiquement parlant, représenter veut dire « rendre présent »; « il y a donc bien une tentative de faire exister, au travers du discours, la réalité dans laquelle on se trouve ${ }^{14}$. En outre, le terme représentations met en relief le caractère dynamique et en évolution d'une idée déterminée. Ces deux caractéristiques marquent une différence par rapport à la notion de stéréotype qu'on verra postérieurement. En ce qui concerne les représentations, il faut ajouter qu'elles sont « le produit d'un travail social collectif, à travers lequel les agents sociaux construisent leurs modes de connaissance de la réalité ${ }^{15}$. Parfois ce qu'on considère comme la réalité est une représentation ou un produit de celle-ci. Cependant, les étudier est utile pour connaître la réalité.

Les représentations fixent les frontières entre le groupe d'appartenance et d'autres groupes. Celles-ci établissent aussi ce qui est proche et compatible avec le groupe. Quand il existe le partage de représentations entre des individus, on dit qu'il y a une adhésion à un groupe et que c'est une façon d'assurer un lien social. Les représentations qu'on a de soi et des autres contribuent énormément à la définition de l'identité d'un groupe. L'étude des représentations de l'étranger est un excellent moyen pour mener à bien une réflexion sur l'identité d'appartenance et sur les étrangers. Il faut insister sur l'idée que les représentations qu'on a de l'autre renvoient surtout à

${ }_{14} \mathrm{~N}$. Auger, "Manuels et stéréotypes", Le français dans le monde, 326 (2003) : 29.
I5 G. Zarate, Représentations de l'étranger et didactiques des langue (Paris : Didier/Crédif, 1995) 29. 
l'identité de l'ensemble d'individus qui les produit. La notion de préjugé se trouve liée aux représentations mais surtout aux stéréotypes, et fréquemment confondue avec ceux-ci.

Même si, originalement le mot préjugé n'était pas porteur d'une signification positive ou négative, son contenu sémantique a souffert une modification. Il « a évolué vers le sens d'un jugement défavorable porté d'avance $»^{16}$. Il fait référence, généralement, à des groupes sociaux pour les dévaloriser. Par conséquent, le préjugé devient un obstacle à la compréhension de l'autre.

Néanmoins, le préjugé n'est pas le seul obstacle. Le stéréotype, étroitement lié à celui-ci et aux représentations, est aussi un facteur à considérer lors de l'enseignement d'une culture étrangère car éviter son étude ou le développer superficiellement pourrait conduire à la fixation ou à la confirmation de certains stéréotypes comme des marques d'une réalité culturelle étrangère. Le mot stéréotype est apparu pour la première fois vers la fin du XVIII ${ }^{\mathrm{e}}$ siècle pour désigner la reproduction d'images imprimées au moyen de formes fixes. En 1922, le journaliste Walter Lippmann introduit ce terme dans les sciences sociales pour affirmer que " la connaissance de la réalité extérieure ne se réalise pas de façon directe, mais par des représentations mentales $\gg{ }^{17}$. En effet, les stéréotypes constituent une réponse mentale, automatique et normale qui se produit avant ou au moment d'entrer en contact avec un étranger ou avec un individu qui ne fait pas partie du groupe auquel on appartient. Cette réponse est influencée par divers facteurs, notamment, l'éducation des parents, les médias, l'école ou les premières impressions qu'on a de l'autre.

Le stéréotype est le résultat d'un processus cognitif qui se manif este à travers une classification et une catégorisation. En effet, l'être humain essaye d'organiser les renseignements qui proviennent de son environnement à partir de plusieurs critères dépendant de ses

\footnotetext{
${ }^{16}$ Auger, 29.

17 M. Di Carlo, L'Interculturel (Paris: Clé International, 1998) 84-85.
} 
besoins et de ses valeurs. Le stéréotype répond surtout à un principe de simplification de la réalité. Face à l'étranger, on essaye de le classer ou le catégoriser par sexe, langue, religion, couleur de la peau, niveau de scolarisation, façon de s'habiller, etc. Ceci dans le but d'associer l' autre à l'information qu'on a stockée à travers le temps et l'influence de quelques facteurs déjà cités et de cette manière prévoir ses comportements et ses modes de penser car suivant le principe de simplification des personnes présentant les mêmes caractéristiques (par exemple ; femmes, arabes, musulmanes, portant de longues robes et un voile, etc.) devaient agir et penser de la même manière.

Il faut dire que ces catégorisations correspondent à une vision du monde qui considère plus important distinguer les individus selon quelques critères plutôt que d'autres. Comme l'a affirmé P. Bourdieu "nous sommes tous des classeurs classés par nos classements ». Ces classements sont, en plus, révélateurs du groupe qui classe plutôt que du groupe qui est l'objet d'une classification, car ils ressortent les filtres culturels au travers desquels se déplacent les perceptions. Ce processus de catégorisation est constitué d'opérations mentales mais il fait partie aussi du contexte social, c'est pour cette raison que c'est au moyen du discours que les individus produisent des jugements sur eux-mêmes et sur les autres.

La langue joue un rôle prépondérant dans ce processus puisque les mots expliquent les schémas mentaux, elle reflète aussi la façon particulière de percevoir le monde et l'expérience individuelle et collective. Bref, chaque fois qu'on s'exprime, on reproduit le propre vécu et les enseignements empruntés à la culture maternelle. Cette dernière idée est très liée à un autre niveau de développement du stéréotype, le niveau identitaire. Comme on l'a mentionné maintes fois, le stéréotype superpose le vécu culturel de l'individu et de la communauté dont il fait partie.

Le stéréotype, comme image de l'altérité, est déterminant pour la formation de l'identité de chacun. On entend par identité l'idée que chaque individu se fait de lui-même et qui comporte son histoire 
personnelle. Cette définition n'est pas le résultat d'une sélection individuelle et délibérée mais le résultat d'un processus à travers lequel l'individu est capable de se connaître et de construire son image par rapport à l'image de son groupe, d'autres groupes et des relations entre les groupes. C'est-à-dire, ce que nous sommes est soumis à l'image que les autres se font de nous, en rapport avec eux et à ce que représentent pour nous les autres.

Il est nécessaire de rappeler que le moyen le plus efficace pour démontrer la spécificité d'une culture, par rapport aux autres, est déterminé par trois facteurs, à savoir, la différence, la séparation et dans la plupart des cas, la dévalorisation de l'autre à travers les stéréotypes. Ceci nous assure que «chacun de nous, pour affirmer son propre moi, est obligé de reconnaître la présence d'un non-moi qui représente à la fois la condition nécessaire et la menace à notre existence $"{ }^{18}$.

Alors, le stéréotype est une réponse appropriée au besoin de faire remarquer la spécificité de la propre culture et une réaction aux sentiments de peur qui peuvent surgir du contact avec l'autre : peur de ne pas pouvoir "protéger» son identité et peur de faire face à l'inconnu. En plus, le stéréotype réduit l'autre à une image rigide, inflexible de même que facile à manipuler et à contrôler et plutôt négative. Tous les individus développent un système personnel de croyances, sur eux-mêmes, sur les autres et sur le monde. Ce système, dans la plupart des cas, coïncide avec celui qu'a établi le groupe dans lequel est immergé l'individu. C'est pourquoi les perceptions, les idées, les croyances ou les valeurs qu'un individu associe à un étranger, sont associées aussi au groupe auquel appartient celui-ci et par conséquent à sa culture.

Mais quelle pourrait être la cause de ce processus qui mène à la formulation d'idées qui se fixent avec le temps dans une société et deviennent des stéréotypes? Pour les anthropologues «l'ethnocentrisme

${ }_{18}$ Di Carlo, 88. 
est le fait de toutes les sociétés humaines, même les plus isolées : la tendance à poser son propre groupe comme mesure absolue à laquelle comparer tous les autres et à juger les diversités comme des infériorités peut être considérée comme universelle ${ }^{19}$.

Il faudrait ajouter que le stéréotype se construit également à un niveau affectif puisqu'on éprouve des sentiments bienveillants ou défavorables à propos d'un système de valeurs différentes, c'est-àdire, envers une culture autre. Ces sentiments sont liés au côté émotionnel de chaque personne et ils dépendent d'une expérience personnelle positive ou négative. C'est pour cette raison que, dans certains cas, un individu appartenant à un groupe social donné, est attiré par les modes de vie d'une culture étrangère et un autre faisant partie du même groupe rejette cette même culture.

Dans le contexte de l'enseignement, toutes ces réflexions théoriques facilitent la construction de l'étude de l'autre. Étant donné que les objectifs de cet enseignement, dans le cadre de l'approche interculturelle, sont de s'approcher de l' autre, de le connaître et surtout de le comprendre, un travail sur les représentations et les stéréotypes s'avère pertinent car il permettrait d'effacer certains préjugés et représentations erronées de l'autre.

En plus, l'étude des stéréotypes mènerait à une réflexion sur la culture maternelle puisqu'en étudiant l'autre, des éléments révélateurs de la culture propre surgiront plus aisément et de cette manière, en même temps qu'on connaît et comprend les comportements des étrangers dans leur contexte, on apprend aussi sur les comportements et façons de penser de la culture maternelle qui, habituellement, vont de soi. Dans ce sens, l'étude des représentations qu'on a des autres et de la culture d'origine se présentera comme un moyen très adéquat pour atteindre ces objectifs dans la classe de FLE.

Cependant, pour avoir du succès, le travail sur les représentations doit se faire à partir d'une lecture de la réalité qui permet d'identifier

${ }_{19}$ Di Carlo, 87. 
quand un fait est un cas particulier et quand il s'agit d'un cas généralisé. De la qualité de cette lecture dépend l'effacement de certaines représentations ou l'affirmation d'autres. Il faut dire qu'une lecture comme telle est le premier pas pour une modification des représentations et mène à une réinterprétation de la réalité de l'étranger.

$\mathrm{Si}$, pour certains spécialistes, le meilleur moyen pour s' approcher de la réalité de l'autre est le contact direct entre les membres de deux communautés dans le cas du Costa Rica, il est nécessaire de penser à une autre manière d'entrer en rapport avec l'étranger. En effet, l'éloignement géographique existant entre notre pays et la France et les autres pays francophones ainsi que les possibilités limitées de voyager dans ces pays ou de contacter des francophones rend difficile cette communication (en dépit des changements positifs de cette situation dans les dernières années). Il existe d'autres possibilités comme le Canada ou les Antilles, toutefois, en ce moment, dans notre contexte, le document authentique devient la méthode la plus performante pour approcher la culture étrangère, ainsi « le quotidien, l'ordinaire et le banal deviennent objets légitimes d'enseignement et le document iconographique fait une entrée en force dans la classe $»^{20}$.

Ce document doit présenter quelques caractéristiques pour le rendre valable ; par exemple pour modifier les représentations, les professeurs doivent disposer des documents permettant de décrire l'inconscient social d'un groupe d'individus.

Il faut tenir compte du fait qu'une culture évolue. Les documents authentiques devraient donc être récents, même si on ne rejette pas les documents d'autres époques puisque ceux-ci pourraient être une source indubitable d'information lorsqu'on veut, par exemple, démontrer une évolution ou une stagnation de quelques stéréotypes.

Parmi ces documents, on trouve les articles de presse, les émissions de télévision ou de radio, des caricatures, les bandes dessinées, les brochures touristiques, les proverbes, les films, et bien

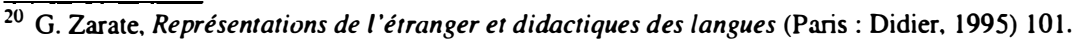


sûr, l'Internet. Ceux-ci véhiculent une vision des étrangers vus par eux-mêmes ou par d'autres étrangers. En outre, ils véhiculent non seulement la pensée, les valeurs et d'autres traits particuliers de l'auteur mais aussi ceux d'une société. Travailler les stéréotypes en s'appuyant sur ces documents permet de développer l'étude de la culture cible à partir du connu ou supposé connu pour aller progressivement vers une connaissance plus approfondie qui conduira à la compréhension de celle-ci.

Les stéréotypes exposeront, aux apprenants, la complexité, la diversité et le caractère dynamique d'une culture et que celle-ci s'exprime à travers divers signes qui peuvent être l'objet de plusieurs interprétations. Dans le cadre d'une approche interculturelle, qui vise une ouverture à l'autre et qui implique un enrichissement mutuel entre les cultures, la prise de conscience des effets des stéréotypes et la mise à plat de ceux-ci, sera essentielle pour atteindre ces objectifs. Dans la classe, l'enseignant doit procurer de conduire l'étude de la culture cible à la découverte de l'autre et faire prendre conscience du risque des généralisations et des interprétations rapides et non fondées. C'est une source de stéréotypes et représentations inexactes.

Véronica Pugibet résume en quelques mots l'importance de l'étude de ces aspects dans une classe de langue et de cultures francophones en abordant les stéréotypes, tant hétéro - qu'auto stéréotypes les étudiants seront amenés à se pencher non seulement sur la culture étrangère, mais aussi sur leur propre culture maternelle. Ils pourront appliquer les outils d'analyse et de réflexion utilisés lors du cours pour approcher leur culture. En effet, il se trouve que les participants d'une culture ne peuvent objectiver, expliquer et systématiser ce qui codifie leur vision du monde. "Cette nouvelle dimension contrastive ne pourra que favoriser une capacité à établir des distinctions et à remettre en cause des évidences ${ }^{21}$.

$\overline{21}$ V. Pugibet, « De l'utilisation des stéréotypes », La civilisation (Paris : Clé International, 1986) 62. 
Dans le milieu costaricien, il est très important de proposer un travail centré sur la culture francophone et non seulement sur la culture française comme on l'a fait traditionnellement dans le but de favoriser l'affirmation du français comme langue à diffusion mondiale ce qui permettrait de montrer la diversité culturelle que représente la francophonie. On rompt ainsi l'image stéréotypée de la langue française. L'approche interculturelle, la langue française et la francophonie partagent des valeurs et des objectifs communs, c'est pourquoi elles sont si compatibles.

Le français, héritage de l'époque coloniale et porteur de valeurs humanistes, une dualité parfois difficile à comprendre mais très réelle, est actuellement enseigné dans presque la totalité des pays du monde. Cette situation lui offre une dimension et une portée internationale. D'ailleurs, cette langue est l'axe principal de l'OIF, une institution qui prône le dialogue des cultures et les échanges culturels, éducatifs, techniques et scientifiques.

Finalement, les nouvelles tendances de l'enseignement du français travaillent sur la nécessité d'accepter les différences, de relativiser les faits culturels dans leur contexte, d'éliminer les obstacles à la compréhension entre les peuples, tels que les stéréotypes; en somme de fomenter la tolérance et le respect envers les autres. 